\title{
Stopping Rules
}

National Cancer Institute

\section{Source}

National Cancer Institute. Stopping Rules. NCI Thesaurus. Code C142698.

A set of circumstances in which the study should be stopped early, keeping in mind the statistical reliability of the circumstances. 